\title{
The Role of Oxidative Stress in the Development of Cognitive Dysfunction Syndrome in Cats. Importance of Antioxidant Prevention and Therapy
}

Cristina Castillo*, Joaquín Hernández

Department of Animal Pathology, Veterinary Faculty, University of Santiago de Compostela, Spain

Received: July 15, 2015; Accepted: September 22, 2015; Published: September 30, 2015

*Corresponding author: Cristina Castillo, Department of Animal Pathology, Veterinary Faculty, University of Santiago de Compostela, Spain, E-mail: cristina.castillo@usc.es

\section{Introduction}

The life expectancy of our pets has increased significantly in recent years, thanks to progress in veterinary medicine, improvement in general hygiene and better nutrition. Aging is characterized by a progressive decline in the efficiency of physiological function and by the increased susceptibility to disease and death. Currently, one of the most plausible and acceptable explanations for the mechanistic basis of aging is the free radical theory of aging. This theory postulates that aging and its related diseases are the consequence of free-radical induced damage to cellular macromolecules and the inability to counterbalance these changes by endogenous antioxidant defenses [1]. The free-radical theory of aging emerged in the mid1950s. This theory suggested that free radicals produced during aerobic respiration have deleterious effects on cell components and connective tissues, causing cumulative damage over time that ultimately results in aging and death. The skepticism first spread around this theory was weakened by the discovery in 1969 of the enzyme Superoxide Dismutase (SOD). The existence of an intracellular enzyme, whose sole function is to remove superoxide anions $\left(\mathrm{O}_{2}^{-} \bullet\right)$, has provided strong biological evidence that free radicals are involved in the aging process. It is now well established that the understanding of aging processes comprises the knowledge of the Oxidative Stress (OS).

Furthermore, the body's ability to maintain homeostasis and adapt to external influences gradually alters with age. Cats age more slowly and, unlike dogs, there are no differences according to breed. Life expectancy of domestic cats is between 14 and 20 years. According to the latest specifications made by FAB (Feline Advisory Bureau, UK) [2] or the American Association of Feline Practitioners, [3], a cat can be considered middle aged when it is between 7 and 10 years old; senior when it is between 11-14 and geriatric from 15 years.

Due to the increased lifespan in pets, ageing biology and pathobiology are emerging as one of the most compelling areas of biomedical research, looking for the early diagnosis of those diseases associated with age, and/or preventive and therapeutic strategies conducted to improve the quality of life for pets when they reach an advanced age. As a general rule, cats 7 years or older - the age when many age-related diseases become more frequently observed - may be considered to be at risk for agerelated problems [4].

As pets age, behavioral changes may be the first indication of declining health and welfare. This is particularly true for some of the most common problems associated with aging, such as pain, sensory decline, and Cognitive Dysfunction Syndrome (CDS). Understanding age-related brain pathology and cognitive dysfunction is essential to fully appreciate the potential value of using biomarkers and/or cognitive status in the future diagnosis and treatment of CDS progression [5].

The aim of this review is to address the role of OS in the pathogenesis of CDS in cats. The currently available laboratory methods for OS assessment will also be discussed, providing reference values of measured oxidants and antioxidants according to the species' age and gender. Finally, we will address the role of different antioxidant strategies for cats, with a preventive or therapeutic use.

\section{Age-related Behavioral Changes}

The increased incidences of age-related diseases often results in behavioral changes [6](Table 1), but is not always related to cognition. Cognition, in terms of animal behavior, refers to mental processes that cannot be observed directly. This includes memory, learning, consciousness and perception [5,7]. These processes allow animals to orient themselves in space, develop habits and recognition and reaction to human family members.

A survey focused on older cats (aged 7-11 years) revealed that $36 \%$ of owners reported behavioral problems in their cats, and this increased to $88 \%$ in cats aged between $16-19$ years. A later study suggested $28 \%$ of pet cats aged $11-14$ years develop at least one geriatric-onset behavior problem, and this increased to more than $50 \%$ for cats of 15 years or older [8].

Taking a human being as a reference, the term dementia encompasses all those pathologies characterized by a state of 
Table 1: Diseases susceptible to cause behavioral changes in senile [6] Arthritis

Primary hypertension or secondary hypertension due to renal failure, diabetes mellitus, acromegaly or hyperadrenocorticism

Hyperthyroidism

Chronic renal failure

Diabetes mellitus

Urinary tract infection

Gastrointestinal disease

Hepatic disease

Neurological disorders (motor or sensory deficits)

Progressive decreased vision or deafness

Brain tumors

Infectious diseases (toxoplasmosis, infectious peritonitis...)

Periodontal disease

Painful processes (in general)

Anxiety and Compulsive disorders

Cognitive dysfunction syndrome

progressive and fatal disrepair of mental faculties [9]. In pets, degenerative dementia affecting brain cortex is called Cognitive Dysfunction Syndrome (CDS) and is defined as an age-related neurodegenerative disorders resulting from a decline in brain functions, including those affecting memory and learning [9-12].

The pathophysiology of CDS is uncertain. Nevertheless, there are pathologic similarities between the brains of humans with Alzheimer's disease and cats with CDS $[13,10]$ : cerebral vascular changes, meningeal thickening, gliosis and ventricular dilatation occur in brains of both diseases. More specifically, the progressive accumulation of a neurotoxic protein called betaamyloid in the brain (in and around neurons) is a consistent feature. These accumulations coalesce to form plaques (neuritic plaques) and are most prominent in the frontal cerebral cortex and in the hippocampus. The beta-amyloid protein, correlates with the severity of cognitive dysfunction in laboratory tests, and can lead to compromised neuronal function, degeneration of synapses, apoptosis-induced neuronal loss, and a depletion of neurotransmitters [14].

In addition to the accumulation of the beta-amyloid protein in the aged feline brain, intraneural accumulation of a hyperphosphorylated microtubule-associated protein (tauprotein) has also been demonstrated [6].

The behavioral changes that can be observed in CDS are grouped into seven categories according to the acronym DISHAAL [5,15-17]:

\section{D- Disorientation/Confusion-Awareness-Spatial orientation}

I- Interactions-Social Relationships

S- Sleep-Wake Cycles; Reversed Day/Night Schedule

H- House soiling (Learning and Memory)

\section{A- Activity-Increased/Repetitive; Apathy/Depressed}

A- Anxiety

L- Learning and Memory-Work, Tasks, Commands

Until recently CDS was a poorly understood syndrome that has been left in the background compared with research into other geriatric diseases. In addition, internal medicine for felines was less studied in comparison with dogs. While a dog's life expectancy once diagnosed the CDS is 1.5 to 2 years [18], the lifespan prognosis for cats has not yet been determined.

Overall, [12] describes one study on cats aged 1 to 3,5 to 9 , and 11 to 16 years assessed locomotor activity, plank walking, reactivity, and spatial reversal learning. The results of that study showed that 1) cats in the oldest group were relatively insensitive to habituation in locomotor sessions, but younger cats became accustomed quickly 2) older cats made fewer mistakes in walking planks than the youngest cats and showed no differences in any neurobiological assessment compared with the other two age groups; 3 ) older cats were more reactive to auditory stimuli than younger cats, and they sustained their reactive responses longer; 4) the oldest cats appeared to have some short-term memory deficits compared with the younger cats.

\section{What is oxidative stress? And what is its role in CDS development?}

All aerobic organisms are susceptible to oxidative stress simply because semi-reduced oxygen species, superoxide and hydrogen peroxide, are produced mainly by mitochondria during respiration. Other sources of free radicals are lysosomes, endoplasmic reticulum, endosomes, peroxisomes and the nucleus $[19,20,21]$.

Mitochondrial DNA is continually exposed to a steady stream of ROS that are generated as by-products during the transfer of electrons to molecular oxygen. The rate of mitochondrial production of ROS increases with age, whereas concentrations of mitochondrial antioxidants decreases. The result is oxidative damage to mitochondrial DNA, with the extent of the damage increasing exponentially with age, which in turn, leads to impaired electron transport and increased leakage of ROS from the electron transport chain, creating a vicious cycle of destruction [22].

Besides oxygen, nitrogen also plays an important role damaging biological molecules via reactive nitrogen species, including both radical and non-radical structures. Reactive Nitrogen Species (RNS) are various nitric oxide-derived compounds, including nitroxyl anion, nitrosonium cation and higher oxides of nitrogen, S-nitrosothiols, and dinitrosyl iron complexes. RNS have been recognized as playing a crucial role in the physiologic regulation of many, if not all, living cells, such as smooth muscle cells, cardiomyocytes, platelets, and nerve and juxtaglomerular cell. They possess pleiotropic properties on cellular targets after both post -translational modifications and interactions with Reactive Oxygen Species (ROS). Elevated levels of RNS have been implicated in cell injury and death by inducing nitrosative stress [23]. 
The exact amount of ROS produced is considered to be about $2 \%$ of the total oxygen consumed during respiration, but it may vary depending on several parameters [24].

Under normal conditions, cells metabolize most of the oxygen into the water without the formation of toxic intermediates. The remainder will be degraded by other means and is responsible for generating free radicals [22]. In situations where there is an increased metabolic activity (e.g. growth stages or inflammatory processes), there is greater tissue $\mathrm{O}_{2}$ demand and part of it are metabolized following the univalent pathway, generating many oxidants [25].

It is worth noting that the term ROS refers to both radicals derived from oxygen, and those which do not derive from it that are generated during the tissue metabolism [26], for this reason ROS are also called Reactive Oxygen Metabolites (ROM). But the oxidants may come from outside either directly or following the metabolism of certain substances. As sources of oxidants we have environmental pollution, sunlight, ionizing radiation, high $\mathrm{O}_{2}$ concentrations, pesticides, heavy metals and certain xenobiotics (chloroform, acetaminophen, and ethanol). Other than mitochondrial respiration, a number of cytosolic enzymes are able to generate ROS. The Nicotinamide Adenine Dinucleotide Phosphate (NADPH) oxidases are a group of plasma membraneassociated enzymes found in a variety of cell types. The function of NADPH oxidases is to produce superoxide from oxygen using electrons from NADPH $[27,28]$. Other endogenous sources of ROS are cytochrome P-450 in the endoplasmic reticulum, peroxisomes, lipoxygenases, cyclooxygenases and xanthine oxidase [22] . Table 2 reflects the main ROS found in mammals.

On the other hand, the body develops defensive mechanisms to counteract the action of ROS: they are the antioxidants. An antioxidant is defined as: Any molecule capable of preventing and/ or avoiding oxidation of another molecule through interaction and stabilization of oxidants; transforming them into more stable molecules with a low reactivity configuration [29]. Antioxidants are classified into three different systems: 1) antioxidant proteins such as albumin, haptoglobin or ceruloplasmin; 2) antioxidant enzymes such as Superoxide Dismutase (SOD), Glutathione Peroxidase (GSH-Px) and Catalase (CAT), and 3) low molecular weight substances such as ascorbate (vitamin C), $\alpha$-tocopherol (vitamin E), glutathione, selenium, uric acid, lycopene and betacarotene among the others.

Intracellular ROS are normally maintained at a low but measurable level within a narrow range, which is regulated

Table 2: Main ROS in the body [35].

\begin{tabular}{|l|l|}
\hline Radicalsa & Non-radicals \\
\hline Superoxide, $\mathrm{O}^{2-}$ & Hydrogen peroxide, $\mathrm{H}_{2} \mathrm{O}_{2}$ \\
\hline Hydroperoxyl radical, $\mathrm{HO}_{2} \cdot$ & Hypochlorous acid, $\mathrm{HClO}$ \\
\hline Hydroxyl radical, $\mathrm{OH}^{\cdot}$ & Ozone $\mathrm{O}_{3}$ \\
\hline Peroxyl radicals, $\mathrm{ROO}^{\cdot}$ & Singlet oxygen, ${ }^{1} \mathrm{O}_{2}$ \\
\hline Alkoxyl radicals, $\mathrm{RO}^{\cdot}$ & \\
\hline
\end{tabular}

by the balance between the rate of production and the rate of scavenging by various antioxidants [30] ROS, at low level under normal conditions, is found to act as signaling molecules in many physiological processes, including redox homeostasis and cellular signal transduction [31]. By activating proteins such as tyrosine kinases, these are important mediators of signal transduction pathways [31]. Dependent on cell types, ROS have been found to function as signaling molecules in cell proliferation, cellular senescence or cell death [32,33]. The divergent effects of ROS on many cellular processes suggest that ROS are not merely detrimental byproducts, but also generated purposefully to mediate a variety of signaling pathways [20].

Nowadays, Oxidative Stress (OS) is considered as a cellular condition induced by the deregulated production of Reactive Oxygen Species (ROS) and Reactive Nitrogen Species (RNS) species, which are highly reactive molecules generated by several biochemical and physiological processes of cellular metabolism under both normal and pathological conditions. The delicate balance between the production and elimination of ROS/RNS (redox homeostasis) determines the normal function of cells [21]. Since OS occurs in almost all diseases, several authors are led to wonder whether OS is part of a disease process, or if it is a disease in itself $[34,35]$. In fact sometimes it's unclear if oxidants trigger the disease or if they are produced as a secondary consequence of the disease and from general tissue damage.

The effects of an OS condition will vary depending on the affected molecule $[22,20]$ :

- If ROS act on lipids, there is lipid peroxidation which is the most frequent oxidative process in the body. The immediate effect is that phospholipid structures of cell membranes (mitochondria, nucleus, etc) are disrupted and destroyed losing their function. In addition, lipid peroxidation generates some highly toxic metabolites.

- If ROS act on proteins there will be alterations in their special conformation and hence, in their function. The most affected proteins are those involved in muscular contractile machinery and enzymes.

- If ROS act on nucleic acids, particularly DNA, they may alter their structure generating a variety of DNA lesions, such as oxidized DNA bases, abasic sites, and DNA strand breaks, which ultimately lead to genomic instability.

- Finally, carbohydrates especially monosaccharides and disaccharides resist the action of oxidants. Glucose counteracts the superoxide radical impairing its action on other molecules. On the other hand, mannose and mannitol are hydroxyl radical scavengers.

Because of their potential to cause oxidative deterioration of DNA, protein, and lipid, ROS have been implicated as one of the causative factors of aging [20]. In feline medicine OS has been involved in kidney or heart disease, diabetes and asthma [36-38], as well as immunocompetence in early ages, parasitic or infectious diseases, or situations that involve stress management such as surgical transplantations [39-41]. 
The brain is considered abnormally sensitive to oxidative damage, and in fact early studies demonstrating the ease of peroxidation of brain membranes supported this notion [24]. In the aging brain, as well as in the case of several neurodegenerative diseases, there is a decline in the normal antioxidant defense mechanisms, which increases the vulnerability of the brain to the deleterious effects of oxidative damage. Aging also is accompanied by changes in membrane fatty acid composition, including a decrease in the levels of Polyunsaturated Fatty Acids (PUFAs) and an increase in monosaturated fatty acids. PUFAs, such as Arachidonic Acid (AA), are abundant in the aging brain and are highly susceptible to free radical attack. Finally, most of the studies conducted to assess the role of protein oxidation in aging brains concluded that there is an increase in oxidized proteins [1].

Focusing on felines, the adult cat brain contains 300 billion neurons, maintained and protected by glial cells. The latter play important roles to support the functions of neurons and nervous system physiology protecting them from ROS, for example, astrocytes are able to release glutathione into the neurons [42]. In general terms, it is assumed that the neurons are more vulnerable than glial to OS and that this phenomenon also affects the cells of the blood-brain barrier [43]. However, the vulnerability of neurons is not only due to their high oxygen demand, but also for their high fat content, low activity of antioxidant enzymes and elevated concentrations of iron and other easily oxidizable substrates such as polyunsaturated fatty acids and catecholamines [6,44]. For cats, the brain damage is translated into the alteration of neuronal proteins (aggregations of protein Tau-hyperphosphorylated) and senile plaques formed by amyloid $\beta$-protein around brain cells [6].

There are few publications focused on the peculiarities of feline CDS, and sometimes the research results obtained in dogs are extrapolated to cats. The study of McCune, et al. [45] showed that brain aging in cats and dogs differs.

In canine aging, frontal lobe volume decreases, ventricular size increases, and there is evidence of meningeal calcification, demyelination, increased lipofuscin, increased apoptotic bodies, neuroaxonal degeneration, and reduced neurons. In cats, age-related pathologies include neuronal loss, cerebral atrophy, widening of sulci, and an increases in ventricular size. Perivascular changes, including microhemorrhage or infarcts in periventricular vessels, are reported in senior dogs and cats, which may contribute to signs of CDS [13].

Comparing Old vs Young cat brains, it has shown a decreased neuronal density in each layer of the cerebellar cortex, denser astrocytes, and fewer neurofilaments-immunoreactive neurons [46]. Diminished cholinergic function is also reported in aged cats. This alteration may contribute to working memory deficits, as well as alterations in motor function and REM sleep. Finally, depositions of $A \beta$ in extracellular plaques and perivascular infiltrates are positively correlated with cognitive impairment in dogs. Nonetheless cats demonstrate more diffuse $A \beta$ plaques [13].
Finally, feline cardiovascular changes that can occur with aging (e.g., anemia, decreased cardiac output and perfusion, systemic hypertension, changes in viscosity) may predispose some cats too, or worsen, brain aging. Older cats can experience infarctions of periventricular vessels. Any such pathology will worsen risks associated with OS. When taken as a whole, these neuropathologic changes put feline brains at the risk of multiple attacks [47].

\section{Current Assessment of Oxidative Stress}

An increasing amount of evidence suggests that OS is linked to the pathophysiology of neurodegenerative diseases. However, definitive evidence for this association has been controversial because of shortcomings found in methods available to assess oxidative stress In vivo. Measuring oxidative stress can be difficult because the biological half-life of free radicals and other reactive species is too short for direct detection. Therefore, evidence has to rely on indirect measurements. These indirect measurements are based on byproducts of oxidative damage to lipids, proteins and DNA, which provide an extensive array of potential biomarkers [21]. The biomarkers that can be used to assess OS In vivo have been an attracting interest, because the accurate measurement of such stress is in need of investigation for its role in lifestyle diseases, as well as to evaluate the efficacy of treatment.

In veterinary medicine, we can assess oS measuring the GSH/GSSH system (reduced glutathione/oxidized glutathione). The first one (GSH) is the primary antioxidant found in cells. It is estimated that over $75 \%$ of cats with chronic diseases have depleted levels of cellular glutathione [48].

Oxidative DNA damage byproducts that are also important markers assessed to evaluate oxidative stress In vivo. $\mathrm{OH} \bullet$ generates a wide range of base and sugar modifications in DNA. However, the initial products of free radical attack undergo transformation into stable end products, whose abundance depends on reaction conditions and more importantly, none of these modifications can identify where the oxidative damage is located [21]. Heaton, et al. [49] described the application of a single-cell gel electrophoresis (comet) assay for assessing levels of DNA damage in canine and feline leukocytes, concluding that the aforementioned comet assay could be a useful tool for determining the optimal effects of dietary antioxidants on a reliable biomarker of oxidative stress, such as cellular DNA status in both species.

Another indicator of OS in felines is the elevation of acute phase protein produced by the liver called serum amyloid A. This indicator of OS and inflammation is similar to C-reactive protein that is often used in dogs to measure inflammation [48].

There are other chemical tests that can be measured in the blood to confirm the presence of OS such as Malondialdehyde (MDA). This is an end-product of free oxygen radical activity that is excreted in urine, blood and other fluids and therefore, serves as a marker of lipid peroxidation [19]. Nevertheless published data for reference values in cats are limited and contradictory in the literature. The presence of normal ranges would permit 
comparison with the abnormal value of these parameters in all diseases accompanied by oxidative stress. Table 3 shows the reference values of Malondialdehyde (MDA), Superoxide Dismutase (SOD) and Catalase (CAT) in male and female cats [19]

The results from this study revealed higher plasma MDA concentrations in male cats than in females. The SOD activity did not show any differences between sexes meanwhile CAT values were lower in males than in females.

In many species, females live longer than males and this may be associated with free radicals which are in lower amounts in the mitochondria of females [50]. The longer lifespan in females may be due to the higher gene expression of antioxidants and the lower oxidative damage of mitochondria in females [51]. Moreover, there is evidence for the strong antioxidant properties of estrogens [52]. The same properties are not characteristic for progesterone and testosterone [53].

Currently, there are several techniques which provide data for the oxidative burden of the body [54-56]. One example is the d-ROM test. This technique has been validated in human beings and many animal species [57-60] and provides information about the oxidant capacity of plasma.
This test determines hydroperoxides, which are breakdown products of lipids, as well as of other organic substrates. Results are expressed as Carratelli Units (CarrU), where $1 \mathrm{CarrU}=0.08$ $\mathrm{mg} \mathrm{H}_{2} \mathrm{O}_{2} / \mathrm{dl}[55]$.

A recent study, Castillo, et al. [61] measured OS in cats and both hydroperoxides and the antioxidant defense system (SAC), estimated by the quantification of the plasma barrier to oxidation. The proportion between pro-oxidants and antioxidants (OSi ratio) was calculated as ROM/SAC. Thus, an increase in the ratio indicates risk of OS due to an increase in ROS production or defensive antioxidant consumption [62]. Tables 4 and 5 show that, the reference values of $\mathrm{d}-\mathrm{ROM}, \mathrm{SAC}$ and OSi in male and female cats, depending on age.

Male cats seem to be subjected to more harmful effects of free radicals and lipid peroxidation as products of cell metabolism. This study concluded with the importance of implementing nutritional strategies within this age range looking for additional antioxidant support, would probably avoid an OS status that predisposes to chronic processes in senior male cats.

\section{Antioxidant Prevention and Therapy}

This should be focused on two aspects: 1) when recommended

Table 3: Reference values of Malondialdehyde (MDA), superoxide dismutase (SOD) and catalase (CAT) in male and female cats [19].

\begin{tabular}{|c|c|c|c|c|c|c|}
\hline Parameter & Units & Sex & Mean value & Minimal value & Maximal value & $\begin{array}{l}\text { Standard } \\
\text { deviation }\end{array}$ \\
\hline \multirow{2}{*}{ Malondialdehyde (MDA) } & \multirow{2}{*}{$\mu \mathrm{mol} / \mathrm{l}$} & Male & 2.317 & 1.822 & 3.133 & 0.376 \\
\hline & & Female & $1.959^{* *}$ & 1.400 & 2.408 & 0.336 \\
\hline \multirow{2}{*}{ Superoxide dismutase (SOD) } & \multirow{2}{*}{$\mathrm{U} / \mathrm{gHb}$} & Male & 4940.791 & 4029.365 & 5910.960 & 632.101 \\
\hline & & Female & 5199.502 & 4477.101 & 6567.729 & 760.180 \\
\hline Catalase (CAT) & $\mathrm{U} / \mathrm{gHb}$ & Male & 9174.578 & 7521.365 & 9593.910 & 603.159 \\
\hline
\end{tabular}

${ }^{*} P<0.05 ;{ }^{* *} P<0.001$ between male and female cats.

Table 4: Mean values ( \pm standard error) of redox balance in cats depending on gender [61].

\begin{tabular}{|c|c|c|c|c|c|}
\hline Parameter & Units & Sex group & Mean value & Minimal value & Maximal value \\
\hline \multirow{2}{*}{$\begin{array}{l}\text { Reactive oxygen metabolites } \\
(\mathrm{d}-\mathrm{ROM})\end{array}$} & \multirow{2}{*}{ (CARR U) } & Female & $123.0 \pm 6.7$ & 78.0 & 148.3 \\
\hline & & Male & $158.8 \pm 13.2$ & 72.0 & 282.0 \\
\hline \multirow{2}{*}{$\begin{array}{l}\text { Serum antioxidant capacity } \\
\text { (SAC) }\end{array}$} & \multirow{2}{*}{$(\mu \mathrm{mol} \mathrm{HClO} / \mathrm{ml})$} & Female & $350.2 \pm 15.6$ & 269.5 & 575.1 \\
\hline & & Male & $377.2 \pm 22.7$ & 288.3 & 664.9 \\
\hline \multirow{2}{*}{ d-ROM/SAC (OSi) } & \multirow[b]{2}{*}{---} & Female & $0.36 \pm 0.02$ & 0.23 & 0.47 \\
\hline & & Male & $0.44 \pm 0.04$ & 0.19 & 0.90 \\
\hline
\end{tabular}

Table 5: Mean values ( \pm standard error) of redox balance in cats depending on age [61].

\begin{tabular}{|c|c|c|c|c|c|}
\hline Parameter & Units & Age group (years) & Mean value & Minimal value & Maximal value \\
\hline \multirow{2}{*}{ Reactive oxygen metabolites (d-ROM) } & \multirow{2}{*}{ (CARR U) } & $2-7$ & $155.9 \pm 13.2$ & 90.6 & 282.0 \\
\hline & & $>7$ and $<12$ & $126.4 \pm 7.6$ & 72.0 & 210.1 \\
\hline Serum antioxidant capacity (SAC) & $(\mu \mathrm{mol} \mathrm{HClO} / \mathrm{ml})$ & $>7$ and $<12$ & $346.8 \pm 13.9$ & 273.4 & 575.1 \\
\hline \multirow{2}{*}{ d-ROM/SAC (OSi) } & \multirow{2}{*}{--} & $2-7$ & $0.43 \pm 0.02$ & 0.19 & 0.90 \\
\hline & & $>7$ and $<12$ & $0.37 \pm 0.04$ & 0.23 & 0.73 \\
\hline
\end{tabular}

The results show that male cats are the population most at risk of OS when compared with females especially between 2 and 7 years of age. 
by the vet, the use of drug therapy, through drugs that minimize the symptoms, improve mental abilities and delay the onset of the disease; 2) the use of dietary management with nutrients or supplements with high antioxidant power.

\section{Drug therapy}

There is no known cure for CDS [10]. Drug treatment should aim to alleviate behavioral problems and cognitive disorders $[14,63]$. For cats, there is no specific medication for treating the CDS. Some studies recommend the administration of sedatives such as benzodiazepines or buspirone [64] or antidepressants such as fluoxetine [6]. While there are no drugs licensed for the treatment of CDS in cats, a number of drugs have been used off-label [8]. These include selegiline, propentofylline and nicergoline, all of which have been used in cats with varying degrees of success [15,65-67].

Here we present the most commonly used drugs in feline medicine:

Propentofylline: Authorized in the UK and other European countries, including France, Germany and Spain, to improve the quality of life in older pets [68]. This drug improves cardiovascular and neurological functions and vitality enhancing cerebral blood flow [10]. The recommended dose for senior cats is $1 / 4$ of a $50 \mathrm{mg}$ tablet daily [5].

Selegiline (also known as deprenyl): Selegiline may alleviate cognitive dysfunction through a number of mechanisms. Release of noradrenaline may be enhanced, and reuptake of noradrenaline may be inhibited. Catecholamine enhancement may lead to improved neuronal impulse transmission. Selegiline metabolites, l-amphetamine and l-methamphetamine may also enhance cognitive function [14]. As a result, this promotes neuronal survival and has a mild antidepressant effect. It is also effective in improving short-term memory; reduces the clinical signs associated with cognitive dysfunction, and improves the quality of life [69]. Selegiline may contribute to a decrease in free radical load in the brain. Selegiline may directly scavenge free radicals and enhance scavenging enzymes such as catalase and superoxide dismutase. The dosage for cats is $0.5-1 \mathrm{mg} / \mathrm{kg}$ once daily $[5,10]$.

Nicergoline: Alkaloid that increases cerebral oxygen consumption and glucose by vasodilation, functioning as neuroprotective. It stimulates mental functions for memory and learning. The suggested dosage for cats is $25-50 \mathrm{mg} / \mathrm{kg}$ orally [8]. Table 6 shows other drugs that may be considered to improve cognitive function or control clinical signs. For each pet, the vet must weigh potential risks against potential benefits.

\section{Nutritional Therapy}

All cats have specific needs for vitamins and minerals, which are normally provided by complete and balanced diets. Approximately one-third of geriatric cats have a reduced ability to digest dietary fats. In these cats, there is a significant correlation between fat digestibility and the digestibility of other essential nutrients, including several B vitamins, vitamin E, potassium, and
Table 6: Doses for drugs for behavior therapy of senior cats [5].

\begin{tabular}{|l|l|}
\hline Oxazepam $^{1}$ & $0.2-0.5 \mathrm{mg} / \mathrm{kg}$ \\
\hline Clonazepam $^{1}$ & $0.02-0.2 \mathrm{mg} / \mathrm{kg}$ \\
\hline Lorazepam $^{1}$ & $0.025-0.05 \mathrm{mg} / \mathrm{kg}$ \\
\hline Diphenhydramine & $1-4 \mathrm{mg} / \mathrm{kg}$ \\
\hline Fluoxetin & $0.5-1.5 \mathrm{mg} / \mathrm{kg}$ once daily \\
\hline Paroxetine & $0.5-1.5 \mathrm{mg} / \mathrm{kg}$ \\
\hline Sertraline & $0.5-1.5 \mathrm{mg} / \mathrm{kg}$ once daily \\
\hline Buspirone & $0.5-1 \mathrm{mg} / \mathrm{kg}$ \\
\hline Trazodone & undetermined \\
\hline Phenobarbital & $2.5 \mathrm{mg} / \mathrm{kg}$ \\
\hline Memantine & undetermined \\
\hline Gabapentin & $5-10 \mathrm{mg} / \mathrm{kg} \mathrm{q} 12$ hours \\
\hline
\end{tabular}

1 Use single dosing prior to sleep or anxiety-evoking event, up to maximum daily dosing for control of ongoing anxiety

other minerals. Thus, older cats should be carefully evaluated for possible nutrient deficiencies and may benefit from supplemental amounts of these nutrients [4].

\section{Supplementation/administration of antioxidants}

During the past 20 years, numerous studies have been conducted to examine several types of interventions to ameliorate oxidative damage. The use of antioxidants to decrease free radical damage may slow cognitive decline and improve the behavioral signs associated with cognitive dysfunction. Antioxidants scavenge and minimize the production of reactive oxygen species, bind metal ions that might make poorly reactive oxygen species more toxic, and repair damage to target tissues [14].

Some of the current treatment strategies appear promising. Most of the treatment strategies used for oxidative stress involve blocking the formation of ROS, scavenging existing ROS, or augmenting endogenous antioxidants. It is likely that the most effective strategies will encompass a combination of treatments that target several steps in the oxidative stress injury cascade (see review of [22]. Antioxidant administration may act through two mechanisms of action: 1) Blocking formation of ROS or 2) Scavenging ROS.

\section{Blocking formation of ROS}

$\mathrm{N}$-acetylcysteine: $\mathrm{N}$-acetylcysteine (NAC) is a chemically modified form of the dietary amino acid cysteine. This enables continued production of glutathione. Burr, et al. [70] found in cats that oral administration at $100 \mathrm{mg} / \mathrm{kg}$ may be effective in the treatment of chronic diseases.

S-Adenosylmethionine (SAMe): This is the methyl donor for biochemical methylation reactions and a precursor of glutathione. SAMe is reported to have hepatoprotective and antioxidant functions. SAMe contributes to the balancing of the activity associated with brain neurotransmitters. It enhances the binding of neurotransmitters to receptor sites within the brain, 
resulting in increased activity of serotonin and dopamine.

In cats, Webb, et al. [71] pointed out that SAMe protects erythrocytes from oxidative damage by limiting Heinz body formation and erythrocyte destruction.

Webb, [72] alerts us to the fact that chemical structure, stability, and oral bioavailability of SAMe depends heavily on the manufacturing process (specific salt used to stabilize the product) and proper packaging (blister pack), and the actual amount of active SAMe in commercially available supplements can be quite variable.

Vitamin E: Interrupts lipid peroxidation. Vitamin E cannot be synthesized by the body, for this reason, it is considered a dietary essential nutrient [73]. This is the vitamin most commonly supplemented in most clinical trials.

A study in cats showed that vitamin E had a mild preventive effect on Heinz body formation and other criteria of oxidative damage due to the consumption of propylene glycol and onion powder [74]. Jewell, et al. [75] described a significant decrease in blood oxidants when cats were supplemented with 540 IU vitamin E/kg of commercial food (Hill's Science Diet, Feline Maintenance).

Vitamin C: Functions as an antioxidant inside and outside the cell. It can be synthesized inside the body of cats and hence is not considered a dietary essential nutrient [73]. Vitamin C helps to maintain oxidative protection and helps to regenerate vitamin $\mathrm{E}$ and glutathione [14]. Vitamin C can act as a pro-oxidant during times of increased amounts of free iron, such as those resulting from inflammation.

Allopurinol: It is a Xanthine Oxidase (XO) inhibitor that blocks the superoxide production generated in the XO system.

Ubiquinol: It donates $\mathrm{H}$ atoms to a radical species and thus removes the radical from any initiation reactions preventing lipid peroxidation. It's oxidized form is ubiquinone (also known as coenzyme Q10).

\section{Scavenging ROS}

$\mathbf{N}$-acetylcysteine: This is a powerful scavenger of hydroxyl radicals and hypochlorous acid.

Fe-chelating agents: Free iron is central to the formation of hydroxyl radicals and many treatment strategies attempts to block iron. Nevertheless, iron chelation treatment can have potentially toxic effects when it interferes with normal iron metabolism. Included in this group, deferoxamine chelates ferrous iron and can reduce ROS injury in animals with experimentally induced conditions.

Dimethyl sulfoxide: Scavenges hydroxyl radicals. The metabolite that forms then traps other ROS. Dimethyl sulfoxide permeates cell membranes to reach intracellular sites of ROS formation. It is also believed that dimethyl sulfoxide inhibits platelet aggregation and increases vasodilation.

Superoxide dismutase: Recently the technology became available to extract non-animal sources of superoxide dismutase enzyme from melon and combine it with gluten (derived from wheat protein) to facilitate transport of the SOD across the gastrointestinal mucosa. A series of studies have now confirmed the bioavailability of this formulation in cats and there are a number of commercially available SOD products for veterinarians to consider [72].

Currently, there is a dietary formula available designed to support a cat's antioxidant and nutritional systems. It contains a Superoxide Dismutase (SOD) complex including SOD, catalase and glutathione peroxidase, in combination with an optimal blend of vitamins (Oxstrin Optimized Feline ${ }^{\odot}$, Nutramax Laboratories, Inc.)

Carotenes: Scavenges ROS increasing glutathione peroxidase activity and works together with vitamin E. Chew, et al. [76] showed that cats are able to readily absorb $\beta$-carotene dosed orally in a water solution, with concentrations of plasma $\beta$-carotene increasing in a dose-dependent manner. Charlton, et al. [77] also showed significant increases in plasma $\beta$-carotene concentrations of cats after feeding a carotenoid-containing diet.

However, early investigations indicated that the domestic cat lacks the ability to convert $\beta$-carotene to vitamin $\mathrm{A}$. The recent interest in carotenoids in pet nutrition as potential antioxidants demands additional studies to clarify the assumptions that dietary $\beta$-carotene cannot be used as a source for vitamin $A$ in domestic cats [78] (Table 7).

Finally, many trace minerals exhibit at least one of their functions in the body by incorporation into antioxidant enzymes. These include selenium (glutathione peroxidase); copper, zinc and manganese (superoxide dismutases) and iron (catalases). These enzymes can all be synthesized in sufficient quantities in the body, provided ample amounts of the mineral are available [73]. Catalase works in conjunction with SOD. Administration of a combination of SOD and catalase conjugate can be effective in attenuating oxidative stress in animals with experimentally induced ischemia-reperfusion injury [22].

Other antioxidants such as ceruloplasmin and ferritin are nonenzymatic antioxidants containing copper and iron, respectively. Currently, trace minerals do not seem to be indicated if the pet is fed a complete and balanced commercial diet, but it is very important if the owner plans to feed it home-made food [79].

\begin{tabular}{|l|l|}
\hline \multicolumn{2}{|l|}{ Table 7: Recommended dosages for antioxidants in cats [22]. } \\
\hline $\begin{array}{l}\text { SAMea } \\
\text { (S-Adenosylmethionine) }\end{array}$ & $\begin{array}{l}20 \mathrm{mg} / \mathrm{kg} / \mathrm{d} \text { as } 1 \text { dose/d or divided into } 2 \\
\text { doses/d; administer orally 0.5 hours before } \\
\text { a meal }\end{array}$ \\
\hline N-acetylcysteine & $\begin{array}{l}50 \mathrm{mg} / \mathrm{kg} \text {; dilute 1:4 with 0.9\% NaCl solution } \\
\text { and administer IV over a 1-h period; can } \\
\text { administer 1 dose every 6 hours }\end{array}$ \\
\hline Vitamin E & 30 units, P0, q 24 hours \\
\hline $\begin{array}{l}\text { Vitamin C (ascorbic } \\
\text { acid) }\end{array}$ & $\begin{array}{l}125 \mathrm{mg}, \mathrm{PO}, \mathrm{q} 12 \text { hours; decrease dosage if } \\
\text { patient develops soft feces }\end{array}$ \\
\hline $\begin{array}{l}\text { Ubiquinone (coenzyme } \\
\text { Q10) }\end{array}$ & $\begin{array}{l}2.0 \mathrm{mg} / \mathrm{kg}, \mathrm{PO}, \mathrm{q} 24 \text { hours. Extrapolated from } \\
\text { dosages used in humans }\end{array}$ \\
\hline
\end{tabular}




\section{Food-based antioxidants}

Dietary supplementation of antioxidants represents the most "palatable" route for this type of therapy. In veterinary medicine particularly in household animals, there are few studies that can serve as a reference given the diversity of the population. However, the initial results indicate that the feed management can be a preventive and therapeutic tool. Nevertheless, there are still questions to resolve such as the adequate nutrients and the dosages $[26,80,11]$.

The logical progression for this sort of research would be to establish the bioavailability and pharmacokinetics of a particular supplement and then move on from there to establish the clinical impact of that supplementation in patients with disease [72].

\section{Plant products}

Plants have been used throughout history for their medicinal properties. This use has often focused on human health, but plants have also been, and still are, applied in veterinary practice and animal health management. All plants produce chemical compounds as part of their normal metabolic activities. These are arbitrarily divided into primary metabolites, such as sugars and fats, found in all plants, and secondary metabolites: Phytobiotics or Phytochemicals, compounds not essential for basic function found in a smaller range of plants [81].

Phytochemicals or plant secondary metabolites are an extremely large group of compounds, of which at least 12,000 have been isolated, a number estimated to be less than $10 \%$ of the total. In many cases, these substances serve as plant defence mechanisms against predation by microorganisms, insects, and herbivores. Some, such as terpenoids, give plants their odors; others (quinones and tannins) are responsible for plant pigment. Many compounds are responsible for plant flavour (e.g., the terpenoid capsaicin from chili peppers), and some of the same herbs and spices used by humans to season food yield useful medicinal compounds.

Compared with synthetic antibiotics or inorganic chemicals, these plant-derived products have unsurprisingly proven to be natural, less toxic, residue free, and are thought to be ideal growth promoters in animal diets [82]. Although there is a global interest in herbal supplementation, there are concerns about the use of untested and unregulated medicines. The scientific community is concerned about the quality, standardization, clinical safety and efficacy of herbal therapies.

They are classified according to the part used (whole plant, root, stem, bark, leaf, flower, fruit and seed), habit (grasses, sedges, herbs, shrubs, climbers and trees), habitat (tropical, sub-tropical and temperate) therapeutic value (antibacterial, antifungal, anti-inflammatory, antiulcer, antioxidant, antiviral, anticancer, immunistimulator etc.) and routes of administration (tincture, decoction, maceration, syrup, inhalation and tisanes), besides the usual botanical classification.

Generally, with respect to biological derivation, formulation, chemical description and purity, phytobiotics comprise of a very wide range of substances and four sub-classes in animal feeding may be categorized: 1) herbs (product from flowering, non-woody and non-persistent plants), 2) botanicals (entire or processed parts of a plant, e.g., root, leaves, bark), 3) essential oils (hydro distilled extracts of volatile plant compounds), and 4) oleoresins (extracts based on non-aqueous solvents) [83].

Nevertheless, it is interesting to take into account the following phytochemical categories based on their chemical properties [84,85]:

\section{Class: Phenolics}

In general, the components with phenolic structures are considered to have stronger antimicrobial activity in comparison with other non-phenolic secondary plant metabolites due to the presence of a hydroxyl group in the phenolic structure.

\section{Subclasses}

\section{Simple phenols and phenolic acids}

These are the simplest bioactive phytochemicals: containing a Hydroxyl Group $(\mathrm{OH})$ attached to a carbon atom that is part of an aromatic ring. Phenols are similar to alcohols but are more soluble in water.

Tea polyphenols, found in the leaves of the tea plant Camellia sinensis have a positive impact on free radicals, limiting the harmful effects of oxidative stress on cell membranes and DNA [79].

Quinones: They are aromatic rings with two ketone substitutions. They are ubiquitous in nature and are characteristically highly reactive.

Flavones and flavonoids: Flavones are phenolic structures containing one carbonyl group (as opposed to the two carbonyls in quinones). In addition to a 3-hydroxyl group yielding a flavonol, Flavonoids, have known properties, such as free radical scavenging activity, inhibition of hydrolytic and oxidative enzyme and anti-inflammatory action [86]. They are found primarily in fruits, vegetables, minimally processed tea (e.g. green tea), and cocoa [22].

The milk thistle plant (Silybum marianum) contains a mixture of bioactive flavonolignans, the most powerful of which is silibinin (also known as silybin). Silybin has been reported to contain antioxidant, anti-inflammatory, and anti-fibrotic properties, which should make it useful for a variety of conditions [72]. A previous study Webb, et al. [87], demonstrated that oral administration of a supplemental silibinin- phosphatidylcholine complex $(10 \mathrm{mg} / \mathrm{kg} / \mathrm{d}$ taken orally for 5 days $)$ increased granulocyte GSH content and phagocytic function, beneficial for cats suffering from diseases associated with oxidative stress.

Tannins: Tannin is a general descriptive name for a group of polymeric phenolic substances that are found in almost every plant part. They are divided into two groups, hydrolyzable and condensed tannins.

Coumarins: These are phenolic substances made of fused benzene and a-pyrone rings. 


\section{Class: Terpenoids (or Isoprenoids) and Essential Oils (EO)}

Terpenoids (or isoprenoids), represent the oldest group of small molecular products synthesized by plants and are probably the most widespread group of natural products. The idea that terpene natural products have important biological functions has taken hold only recently, and there are considerable difficulties in testing these compounds in natural settings [88]. Many terpenes are hydrocarbons, but oxygen-containing compounds such as alcohols, aldehydes or ketones are also found. These derivatives are frequently named terpenoids.

The terpenoid capsaicin (8-methyl-n-vanillyl-6-nonenamide), a pungent component found in red pepper (Capsicum annuum) can prevent oxidative stress when consumed in a short period (weeks). It has been found that capsaicin is able to enhance SOD, catalase and glutathione activities [89].

Allicin is an oily extract that can be found in garlic and onions and is toxic for cats [90]. The breakdown of allicin produces the toxic component n-propyl disulfide which causes oxidative damage to hemoglobin and erythrocytes membranes, resulting in the formation of metahemoglobin, Heinz bodies, leading to intravascular hemolysis and anemia.

Mono- and sesqui terpenes are the chief constituents of the EO while the other terpenes are constituents of balsams, resins, waxes, and rubber [88]. The fragrance of plants is carried in the essential oil fraction. These oils are secondary metabolites that are highly enriched in compounds based on an isoprene structure.

Essential oils are mixtures of terpenes or phenylpropane derivatives in which, the chemical and structural differences between compounds are minimal [91]. Nevertheless, phenolic compounds possessing a C3 side chain at a lower level of oxidation and containing no oxygen are classified as EO and often cited as antimicrobial as well [84].

Saponins are an important class of plant metabolites that show enormous structural diversity. Chemically, saponins are high-molecular-weight glycosides in which sugars are linked to a triterpene or steroidal aglycone moiety [92]. Saponins can be found in most vegetables, beans and herbs. The bestknown sources of saponins are peas, soybeans, and some herbs with names indicating foaming properties such as soapwort (Saponaria officinalis), soaproot (Chlorogalum pomeridianum), soapbark (Quillaja saponaria) and soapberry (Sapindus saponaria Sapindus oahuensis). Commercial saponins are extracted mainly from Yucca schidigera, Quillaja saponaria and Panax Ginseng.

Ginseng stem-leaf saponins (Saponaria officinalis) significantly inhibited cyclophosphamide-induced oxidative stress by increasing total antioxidant capacity, and the levels of glutathione, ascorbic acid, and $\alpha$-tocopherol, while elevating the activity of total superoxide dismutase, catalase, and glutathione peroxidase, as well as decreasing the protein carbonyl content and malondialdehyde [93].

\section{Class: Alkaloids}

These are heterocyclic nitrogen compounds. Methylxanthines are plant alkaloids that include theobromine, caffeine, and theophylline. These can be found in a variety of products, such as the cacao bean or tea leaves. Methylxanthines are toxic for cats [90].

It is worth noting that there is a strong tendency to supplement diets with antioxidants of plant origin. Nevertheless, more clinical tests need to be conducted to comprehensively understand the effects on pets health and to determine the adequacy of each phytochemical in the pet's conditions.

\section{Other Dietary Components}

Taurine, an amino acid, is a critically important nutrient for cats. Its benefit is for successful reproduction, healthy eyesight and heart function. Though important to cats, taurine is not similarly essential for dogs. Taurine is beneficial for both species as an antioxidant, known to protect cell membranes from damage [14].

Adequate dietary protein in elder cats is critical to support endogenous glutathione production, a key antioxidant for disease prevention [4].

The combination of L-carnitine and $\alpha$-lipoic acid improves the neural mitochondrial function in geriatric animals, and also has a synergistic effect on the memory improvement [25].

There are also phosphatidylserine, a natural phospholipid. Its effect consists of maintaining cell activity by promoting the function of the plasma membrane. Clinical studies conducted in humans and experimental animals showed a clear improvement in aspects related to learning and memory.

Conjugated Linoleic Acid (CLA) is an essential fatty acid which cannot be synthesized in the body. It is found naturally in foods of animal origin (meat and dairy) and some vegetables (corn). Some studies suggest its potential use as an antioxidant, although information on this is still scarce $[94,95]$.

The National Research Council (2006) recommended that all feline diets contain a minimum of $1 \mathrm{~g}$ of linoleic acid per 1000kcal diet. The Association of American Feed Control Officials Nutrient Profile for Cats lists the dietary requirement for linoleic acid in growing and adult cats as $1.25 \mathrm{~g} / 1000 \mathrm{kcal}$ [96].

Marine oils are especially rich in the desired long chain n-3 fatty acids, and because of this they are the preferred dietary source for cats, given that these animals are unable to efficiently convert the shorter $\mathrm{n}-3$ fatty acids contained in vegetable oils such as flax [97].

There are commercial diets that include a wide range of antioxidants and free radical scavengers (eg $\mathrm{N}$-acetylcysteine, $\alpha$-lipoic acid, vitamins C and E, L-carnitine, coenzyme Q10 and phosphatidylserine). They also include essential fatty acids such as docosahexaenoic acid (DHA) or eicosapentaenoic acid (EPA).

The clinical signs of CDS in dogs have also been reduced by feeding a diet supplemented with plant-derived Medium-Chain Triglycerides (MCTs), which provide ketones as a more efficient energy source for the brain. Unfortunately, cats are generally not 
keen on eating diets enriched with MCTs so it is unclear if this approach will be useful for cats with CDS $[5,8]$.

Gunn-Moore, [6] describes the study conducted on 90 cats aged between 7 and 17 years old, fed a commercial diet (Purina Nestlé Age 7+) and supplemented with antioxidants (vitamin E and beta-carotene), essential fatty acids (omega 3 and 6) and chicory root (containing inulin, regulator of intestinal flora). The results showed a better quality of life (in terms of overall health and cognition) compared with those who had not been supplemented.

Results from Cupp, et al. [96] study suggested that a diet supplemented with a combination of antioxidants (vitamin $\mathrm{E}$ and carotenes), prebiotic, and long-chain polyunsaturated fatty acids, at specifically formulated levels, can increase lifespan in senior cats.

Senior cats fed a diet containing supplemental antioxidants vitamin $\mathrm{E}$ and $\beta$-carotene, dried chicory root, and a blend of n-3 and n-6 fatty acids lived significantly longer than cats fed a standard nutritionally complete feline diet.

Currently, the market provides a wide variety of diets that combine the effects of antioxidants, mitochondrial cofactors (L-carnitine, DL- $\alpha$-lipoic acid), plant extracts, and/or essential fatty acids, in an attempt to improve cognitive function and delay cognitive decline.

The study of Pan, et al. developed in 2013 (quoted by GunnMoore, [8] demonstrated that middle-aged and older cats fed a diet supplemented with a combination of fish oil, antioxidants, arginine and key B-vitamins, were found to have enhanced brain functions compared to those fed a control diet.

\section{Conclusions}

Specific clinical trials are needed to design helpful therapeutic interventions in CDS cats. However, the first step is to obtain a reliable tool enabling early diagnosis of the disease. Hitherto, the evaluation of oxidative stress may be considered more as a preventative assessment than a diagnostic tool. Even so, the evaluation of oxidative stress and its factors of variation with age allow us to monitor the success - or failure - of the many nutritional and pharmacological strategies that exist in Feline Medicine today.

\section{References}

1. Gemma C, Vila J, Bachstetter A, Bickford PC. Oxidative Stress and the Aging Brain: From Theory to Prevention. In: Brain Aging: Models, Methods, and Mechanisms. Riddle DR. (ed.). Boca Raton (FL): CRC Press; 2007.

2. Feline Advisory Bureau (FAB, UK). Well Cat for Life. http://icatcare. org/vets/wellcat-life. (accessed June 9, 2010).

3. Pittari J, Rodan I, Beekman G, Gunn-Moore D, Polzin D, Taboada J, et al. American Association of Feline Practitioners. Senior Care Guidelines. J Fel Med Surg. 2009;11:763-778.

4. Laflamme DP. Nutrition for aging cats and dogs and the importance of body condition. Vet Clin North Am Small Anim Pract. 2005;35(3):713742 .
5. Landsberg GM, Nichol J, Araujo JA. Cognitive Dysfunction Syndrome. A disease of canine and feline brain aging. Vet Clin North Am Small Anim Pract. 2012;42(4):749-768. doi: 10.1016/j.cvsm.2012.04.003.

6. Gunn-Moore D. Geriatric cats and cognitive dysfunction syndrome. Proceedings of the WSAVA Congress, Dublin, Ireland. 2008.

7. McFarland D. The Oxford companion to animal behaviour. Oxford, UK: Oxford University Press; 1987.

8. Gunn-Moore D. Dementia in geriatric cats. Vet Focus. 2014;25(1):1416.

9. Azkona G, García-Belenguer S, Unzueta A, Chacón G, Rosado B, León M, Palacio J. (2005). El síndrome de disfunción cognitiva en el perro geriátrico. Pequeños Animales. 56:31-41.

10. Dewey CW. A practical guide to canine and feline neurology. 2nd. Edition. Iowa, USA: Blackwell Publishing; 2008.

11. Head E, Refina J, Zicker S. Oxidative Stress, Aging, and Central Nervous System Disease in the Canine Model of Human Brain Aging. Vet Clin North Am. 2008;38(1):167-178.

12. Overall KL. Manual of clinical behavioural medicine for dogs and cats. Canada: Elsevier-Mosby; 2013.

13. Brellou G, Papaioannou N, Lekkas S, Vlemmas I. Age related changes in the brain of cat. Proc. 29th WSAVA Congress, Greece. 2004.

14. Landsberg GM, Head E. Aging and effects on behaviour. In: Hoskins JD. Geriatrics and gerontology of the dog and cat. 2nd ed. Saunders. 2004. 29-42.

15. Landsberg GL, Hunthausen W, Ackerman L. The effects of aging on behavior in senior pets. In: Handbook of behavior problems in the dog and cat. 2nd ed. London: WB Saunders;2003.269-304.

16. Heath S. Canine dementia. Its diagnosis, treatment and medical differences. Proceedings of 29th WSAVA Congress, Greece. 2004.

17. Landsberg G, Hunthausen W, Ackerman L. Handbook of Behavior Problems of the Dog and Cat. 2nd ed. China; Saunders;2004

18. Heiblum M, Labastida R, Chavez G, Tejeda A. "Didy," a clinical case of cognitive dysfunction syndrome. J Vet Behav. 2007;2:68-72.

19. Todorova I, Simeonova G, Kyuchukova D, Dinev D, Gadjeva V. Reference values of oxidative stress parameters (MDA, SOD, CAT) in dogs and cats. Comp. Clin. Path. 2005;13:190-194.

20.Cui H, Kong Y, Zhang H. Oxidative Stress, mitochondrial dysfunction, and aging. J. Signal Transduct. 2012;2012:646354. doi: $10.1155 / 2012 / 646354$.

21. Navarro-Yepes J, Zavala-Flores L, Annadurai A, Wang F, Skotak $\mathrm{M}$, Chandra $\mathrm{N}$, et al. Antioxidant gene therapy against neuronal cell death.Pharmacol. Ther. 2014;142(2):206-230. doi: 10.1016/j. pharmthera.2013.12.007.

22. McMichael M. Oxidative stress, antioxidants, and assessment of oxidative stress in dogs and cats. J Am Vet Med Ass. 2007;231(5):714720.

23. Martínez MC, Andriantsitohaina R. Reactive nitrogen species: molecular mechanisms and potential significance in health and disease. Antioxid Redox Signal. 2009;11(3):669-702.

24. Floyd RA, Hensley K. Oxidative stress in brain aging: Implications for therapeutics of neurodegenerative diseases. Neurobiol. Aging. 2002;23(5):795-807.

25. Pereira V, Guimarey R, Hernández J, Benedito JL, García Vaquero 
M, Vázquez P, Castillo C. Oxidative stress and cognitive dysfunction in senior cats and senile. Veterinary Consultation Broadcasting. 2011;183:45-52.

26. Huerta M, Ortega ME, Cobos M, Herrera JG, Díaz-Cruz A, Guinzberg R. Oxidative stress and the use of antioxidants in domestic animals Interscience. 2005;30(12):728-734.

27. Finkel T, Holbrook NJ. Oxidants, oxidative stress and the biology of ageing.Nature. 2000;408(6809):239-247.

28. Lambeth JD. NOX enzymes and the biology of reactive oxygen.Nature Rev. Immunol. 2004;4(3):181-189.

29. Halliwell B, Gutteridge JMC. Free Radicals in biology and medicine. 4th Edition. Oxoford University Press, UK. 2007.

30. Sies H. Strategies of antioxidant defense. Eur J Biochem. 1993;215:213 219.

31. Dröge W. Free radicals in the physiological control of cell function. Physiol Rev. 2002;82(1):47-95

32. Clément MV, Pervaiz S. Reactive oxygen intermediates regulate cellular response to apoptotic stimuli: a hypothesis. Free Rad Res. 1999;30(4):247-252

33. Lundberg AS, Hahn WC, Gupta P, Weinberg RA. Genes involved in senescence and immortalization. Curr Opin Cell Biol. 2000;12(6):705709 .

34. Mandelker L. Cellular effects of common antioxidants. Vet Clin North Am Small Anim Pract. 2008;38(1):199-211. doi: 10.1016/j. cvsm.2007.11.002.

35. Castillo C, Wittwer F, Cerón JJ. Oxidative Stress in Veterinary Medicine. Vet Med Int. 2011;812086. doi: 10.4061/2011/812086.

36. Harper EJ. El posible uso terapéutico de los antioxidantes en las enfermedades clínicas. Waltham Focus. 2000:10:12-16.

37. Webb CB, Falkowsky L. Oxidative stress and innate immunity in feline patients with diabetes mellitus: The role of nutrition. J. Fel Med Surg. 2009;11(4):271-276. doi: 10.1016/j.jfms.2008.07.004

38. Christiansen LB, Dela F, Koch J, Hansen CN, Leifsson PS, Yokota T. Impaired cardiac mitochondrial oxidative phosphorylation and enhanced mitochondrial oxidative stress in feline hypertrophic cardiomyopathy. Am J Physiol Heart Circ Physiol. 2015;308(10):H1237-1247.

39. Koelsch S, Smith B. Fortaleciendo las barreras contra las infecciones felinas: Los beneficios de las dietas enriquecidas con antioxidantes. Waltham Focus. 2001.11:32-33.

40. Halling KB, Ellison GW, Armstron D, Aoyagi K, Detrisac CJ, Graham JP, Newell SP, Martin FG, Van Gilder J.M. Evaluation of oxidative stress for the early diagnosis of allograft rejection in feline renal allotransplant recipients with normal renal function.Can Vet J. 2004;45(10):831-837.

41. Kayar A, Dokuzeylul B, Kandemir FM, Kirbas A, Bayrakal A, Or ME. Total oxidant and antioxidant capacities, nitric oxide and malondialdehyde levels in cats seropositive for the feline coronavirus. Veter Medicina. 2015;60(5):274-281.

42. Testa MP, Alvarado O, Wournell A, Lee J, Guilford FT, Henriksen SH Phillips TR. Screening Assay for Oxidative Stress in a Feline Astrocyte Cell Line, G355-5. J Vis Exp. 2011;13(53):e2841. doi:10.3791/2841.

43. Cerdá C, Borrego S, Sáez G. Estrés Oxidative in neurodegenerative diseases . Monographs of the Royal Academy of Pharmacy, 2010;29:283-301.
44. Onyango IG, Shaharyar MK. Oxidative Stress, mitochondrial dysfunction, and Stress signalling in Alzheimer's disease. Curr Alzheimer Res. 2006;3(4):2-11.

45. McCune S, Stevenson J, Fretwell L, Thompson A, Mills DS. Ageing does not significantly affect performance in a spatial learning task in the domestic cat (Felis silvestris catus). Appl. Anim. Behav. Sci.112(34):345-356

46.Zhang C, Hua T, Zhu Z, Luo X. Age-related changes of structures in cerebellar cortex of cat. J. Biosci. 2006;31(1):55-60.

47. Overall KL. Assessing brain aging in cats. 2012. http://veterinarynews. dvm360.com. (accessed August 17, 2015).

48. Mandelker L. Oxidative stress, free radicals and cellular damage. In: Studies on Veterinary Medicine. Mandelker L. Vajdovich P. (ed.). New York: Humana Press-Springer;2011.

49. Heaton PR, Ransley R, Charlton CJ, Mann SJ, Stevenson J, Smith BHF, et al. Application of a single-cell gel electrophoresis assay (Comet) for assesing levels of DNA damage in canine and feline leukocytes.J. Nutr. 2002;132(6):1598S-1603S

50.Sastre J, Borras C, Garcia-Sala D, Lloret A, Pallardo FV, Vina J. Mitochondrial damage in aging and apoptosis. Ann Ann N Y Acad Sci. 2002;959:448-451.

51. Borras C, Sastre J, Garcia-Sala D, Lloret A, Pallardo FV, Vina J. Mitochondria from females exhibit higher antioxidant gene expression and lower oxidative damage than males. Free Radic Biol Med. 2003;34(5):546-552.

52.Tudus PM. Estrogen and gender effects on muscle damage, inflammation, and oxidative stress. Can. J. Appl. Physiol. 2000;25(4):274-287.

53. Barp J, Araujo AS, Fernandes TR, Rigatto KV, Llesuy S, Bello-Klein A, et al. Myocardial antioxidant and oxidative stress changes due to sex hormones. Braz. J. Med. Biol. Res. 2002;35(9):1075-1081.

54.Alberti A, Bolognini L, Macciantelli D, Carratelli, M. The radical cation of $\mathrm{N}, \mathrm{N}$-diethylpara-phenylendiamine: a possibile indicator of oxidative stress in biological samples. Res. Chem. Intermediates. 2000;26(3):253-267.

55. Trotti R, Carratelli M, Barbieri M. Performance and clinical application of a new, fast method for the detection of hydroperoxides in serum. Panminerva Med. 2000;44(1):37-40.

56. Vassalle C, Boni C, Di Cecco P, Ndreu R, Zucchelli GC. Automation and validation of a fast method for the assessment of in vivo oxidative stress levels. Clin. Chem. Lab. Med. 2006;44(11):1372-1375.

57. Oriani G, Corino C, Pastorelli G, Pantaleo L, Ritieni A, Salvatori G. Oxidative status of plasma and muscle in rabbits supplemented with dietary vitamin E. J Nutr Biochem. 2001;12(3):138-143.

58. Ballerini A, Civitareale C, Fiori M, Regini M, Brambilla G. Traceability of inbred and crossbred Cinta Senese Pigs by evaluating the oxidative stress. J Vet Med A Physiol Pathol Clin Med. 2003;50(3):113.

59. Brambilla G, Ballerini A, Civitareale C, Fiori M, Ner B, Cavallina R, et al Oxidative stress as a bio-marker of estrogen exposure in healthy veal calves. Analytica Chimica Acta. 2003;483:281-288.

60. Sivaram AG, Suresh MV, Indira M. Combined effect of ascorbic acid and selenium supplementation on alcohol-induced oxidative stress in guinea pigs. Comp Biochem Physiol C Toxicol Pharmacol. 2003;134:397-401

61. Castillo C, Pereira V, Abuelo A, Guimarey R, García Vaquero M, 
Benedito JL, et al. Preliminary results in the redox balance in healthy cats: influence of age and gender. J Feline Med Surg. 2012a;15(4): 328332. doi: $10.1177 / 1098612 X 12467996$.

62. Abuelo A, Hernández J, Benedito JL, Castillo C. Oxidative stress index (OSi) as a new tool to assess redox status in dairy cattle during the transition period. Animal. 2013;7(8):1374-1378. doi: 10.1017/ S1751731113000396.

63. Ibáñez M, Anzola B, Valverde C. Senile dementia in a dog: Case report. Caniset Felis. 2010;(102):106-111.

64. Crowell-Davis S.L. Cognitive dysfunction in senior pets. Compend Contin Educ Vet. 2008;30(2):106-108.

65. Landsberg G, Araujo JA. Behavior problems in geriatric pets. Vet Clin North Am Small Anim Pract. 2005;35(3):675-698.

66. Studzinski CM, Araujo JA, Milgram NW. The canine model of human cognitive aging and dementia: pharmacological validity of the model for assessment of human cognitive-enhancing drugs. Prog Neuropsychopharmacol Biol Psychiatry. 2005;29(3):489-498.

67. Landsberg G. Therapeutic options for cognitive decline in senior pets. J Am Anim Hosp Assoc. 2006;42(6):407-13.

68. Scaramuzzi RJ, Baker DJ. Possible therapeutic benefits of adenosinepotentiating drugs in reducing age-related degenerative disease in dogs and cats. J Vet Pharm. Ther. 2003;26(5):327-335.

69. Siwak CT, Callahan H, Milgram NW. Adrafinil: effects on behavior and cognition in aged canines. Prog Neuropsychopharmacol Biol Psychiatry. 2000;24:709-726.

70. Buur JL, Diniz PP, Roderick KV, KuKanich B, Tegzes JH Pharmacokinetics of $\mathrm{N}$-acetylcysteine after oral and intravenous administration to healthy cats. Am J Vet Res. 2013;74(2):290-293.

71.Webb CB, Twedt DC, Fettman MJ, Mason G. S-adenosylmethionine (SAMe) in a feline acetaminophen model of oxidative injury. J Fel Med Surg. 2003;5(2):69-75.

72. Webb CB. Silybin and antioxidants.Western Veterinary Conference. Available at: http://www.wvc.org/images/session_notes_2013.

73. NRC. Nutrient requirements of dogs and cats. National Research Council of the National Academies. Washington, USA: The National Academies Press;2003.

74. Hill AS, O'Neill S, Quinton MA, Rogers R, Christopher MM. Antioxidant prevention of Heinz body formation and oxidative injury in cats. Am J Vet Res. 2001;62(3):370-374.

75. Jewell DE, Toll PW, Wedekind KJ, Zicker SC. Effect of increasing dietary antioxidants on concentrations of vitamin $\mathrm{E}$ and total alkenals in serum dogs and cats. Vet Ther. 2000;1(4):264-272.

76. Chew BP, Park JS, Weng BC, Wong TS, Hayek MG, Reinhart GA. Dietary $\beta$-carotene absorption by blood plasma and leukocytes in domestic cats.J. Nutr. 2000;130(9):2322-2325.

77. Charlton CJ, Harper EJ, Mazur A. Dietary carotenoid absorption in the domestic cat. FASEB J. 2000;14(4):A519- 363.15

78. Schweigert FJ, Raila J, Wichert B, Kienzle E. Cats absorb $\beta$-Carotene, but it is not converted to Vitamin A. J Nutr. 2002;132(6):1610S-1612S.

79. Wortinger A, Burns KM. Nutrition and Disease management for veterinary technicians and nurses. John Wiley \& sons, UK. 2015.

80. Chan DL. The role of nutrients in modulating diseases. J Small Anim Pract. 2008;49(6):266-71. doi: 10.1111/j.1748-5827.
81. Castillo C, Hernandez J, Pereira V, Benedito JL. Update about nutritional strategies for preventing ruminal acidosis. In: Advances in Zoology Research. OP Jenkins, Ed., New York, NY, USA: Nova Science Publishers;2012b. p.1-84.

82. Hashemi SR, Zulkifli I, Hair Bejo M, Farida A, Somchit MN. Acute toxicity study and phytochemical screening of selected herbal aqueous extract in broiler chickens. Int J Pharmacol. 2008;4:352-360.

83. Hashemi SR, Davoodi H. Herbal plants and their derivatives as growth and health promoters in animal nutrition. Vet Res Comm. 2011;35(3):169-180. doi: 10.1007/s11259-010-9458-2.

84. Cowan MM. Plant Products as Antimicrobial Agents. Clin Microbiol Rev. 1999;12(4):564-82.

85. Rochfort S, Parker AJ, Dunshea FR. Plant bioactives for ruminant health and productivity. Phytochemistry. 2008;69(2):299-322.

86. Atanassova, M, Georgieva S. Comparative polyphenol composition and antioxidant capacity of the bulgarian plants (dry herbs). Electronic J. Environ. Agric. Food Chem. 2010;9(9):1514-1523.

87. Webb CB, McCord KW, Twedt DC. Assessment of oxidative stress in leukocytes and granulocyte function following oral administration of a silibinin-phosphatidylcholine complex in cats. Am. J Vet Res. 2009;70(1):57-62. doi: 10.2460/ajvr.70.1.57.

88. Gershenzon J, Dudareva N. The function of terpene natural products in the natural world. Nat Chem Biol. 2007;3(7):408-414

89. Srivastava SK. Role of Capsaicin in Oxidative stress and cancer.. TX, USA: Springer Amarillo;2013.

90. Gugler K, Piscitelli C, Dennis J. Hidden dangers in the kitchen: Common foods toxic to dogs and cats. Compend Contin Educ Vet. 2013;35(7):E2.

91. Oprean R, Oprean L, Tamas M, Sandulescu R, Roman L. Essential oils analysis. II. Mass spectra identification of terpene and phenylpropane derivatives. J Pharm Biomed Anal. 2001;24(5-6):1163-1168.

92. Wallace RJ. Antimicrobial properties of plant secondary metabolites. Proc Nutr Soc. 2004;63(4):621-629.

93. Yu J, Chen Y, Zhai L, Zhang L, Xu Y, Wang S, et al. Antioxidative effect of ginseng stem-leaf saponins on oxidative stress induced by cyclophosphamide in chickens. Poult Sci. 2015;94(5):927-933. doi: 10.3382/ps/pev055.

94. Cruz S, de Juan-García P, Sánchez FG. CLA antioxidants and prooxidant? Fats and oils. 2000;51(4):268-274.

95. Chan DL. The role of nutrients in modulating diseases. J Small Anim Pract. 2008;49(6):266-71. doi: 10.1111/j.1748-5827.2008.00589.x.

96. Cupp CJ, Jean-Philippe C, Kerr WW, Patil AR, Perez-Camargo G. Effect of Nutritional Interventions on Longevity of Senior Cats. Intern J Appl Res Vet Med. 2006;4(1):34-50.

97. Fascetti A, Delaney SJ. Applied veterinary clinical nutrition. Ed. WileyBlackwell. Oxford, UK: John Wiley \& Sons;2012. 